\title{
Feminismo, academia y cambio social
}

Mirta González Suárez

Resumen: El feminismo, como teoría y práctica social, analiza y realiza propuestas para superar el patriarcado en todos los ámbitos y entre ellos el académico donde se insertan sus núcleos principales tales como el sexismo, la separación bipolar entre lo masculino y femenino, el poder, el control del cuerpo de las mujeres y la violencia.
El feminismo es una teoría ampliamente desarrollada en la academia (ver Amorós, Celia "Historia de la Teoría Feminista, 1994), pero también implica una posición política de defensa de los derechos de las mujeres.

Entendemos por feminismo entonces tanto una posición teórica como una propuesta social. En Costa Rica una variada presentación sobre las diversas opciones del feminismo se resume en el libro "Feminismo en Costa Rica?" (Aguilar, Lorena et $a l .$, 1995). A nivel internacional Descarries, Belanger y Roy, Shirley (1992) compilan las principales tendencias de acuerdo con los objetivos centrales, las que van desde la valoración de los roles asignados a las mujeres al cambio de la estructura de poder.

Ante tal amplitud propositiva, sin embargo, permanecen elementos centrales en la teoría, a saber: El patriarcado es una de las formas principales de organización del poder social, que se caracteriza por utilizar esquemas intrincados de explotación, mantenida por la violencia, sea esta abierta o encubierta. Dos de los pilares más importantes de control patriarcal es la creencia de que hombres y mujeres son esencialmente diferentes y el establecimiento de una mayor valoración de los roles sociales asignados a los varones. 
La propuesta social del feminismo es la eliminación del patriarcado, con lo que hombres y mujeres podrán relacionarse como seres humanos en igualdad de condiciones, dejando atrás estructuras de violencia.

Desde el feminismo se han develado numerosos aspectos del poder patriarcal, algunos de los cuales se presentan a continuación:

\section{Ideología Patriarcal}

\section{Esferas separadas: lo femenino y lo masculino}

El principal mito del patriarcado es explicar las diferencias conductuales entre hombres y mujeres como resultado de la fisiología de cada sexo. Al considerar que hombres y mujeres son seres fundamentalmente distintos y por eso se comportan en forma diferenciada, algunas de las dicotomías más comunes son:

$\begin{array}{ll}\text { Hombres } & \text { Mujeres } \\ \text { Fuertes } & \text { Débiles } \\ \text { Activos } & \text { Pasivas } \\ \text { Dominantes } & \text { Sumisas } \\ \text { Agresivos } & \text { Pacíficas } \\ \text { Intelectuales } & \text { Emocionales } \\ \text { Independientes } & \text { Dependientes } \\ \text { Objetivos } & \text { Subjetivas } \\ \text { Competitivos } & \text { Cooperadoras } \\ \text { Distanciados } & \text { Cariñosas, etc. }\end{array}$

El objetivo central es presentar esta situación como "lo natural" -y por lo tanto validar su propuesta de que los hombres son- y deben ser los que toman las decisiones importantes y las mujeres dependen de ellos.

Propuesta feminista: Hombres y mujeres son educados de diversas maneras y sus conductas son igualmente diversas. El comportamiento de las personas no es asignado por la biología -y por lo tanto incambiable- sino que cada ser debe tener opciones y alternativas conductuales. Lo que caracteriza a los seres humanos es su socialización y diversidad.

\section{El poder social}

Para acceder al análisis del patriarcado es necesario adentrarse en la estructura del poder social, del cual las mujeres son sistemáticamente -si no excluidas- educadas para propagar la ideología patriarcal.

La responsabilidad social de las mujeres se concentra en la casa mientras que la esfera pública se considera un ámbito fundamentalmente masculino.

Propuesta feminista: las mujeres tienen tanto derecho como los hombres a participar en la toma de decisiones en todos los niveles.

\section{Sexismo o discriminación por género}

Se produce discriminación cuando no se permite, por el sólo hecho de pertenecer a un grupo específico, desarrollar las potencialidades. La discriminación se puede ejercer en forma conciente o inconciente y su existencia se observa en los resultados diferenciados. 
En el patriarcado el sexismo se presenta en dicotomías que se asumen como propias y naturales para hombres o mujeres, tales como las siguientes:

$\begin{array}{ll}\text { Mujeres } & \text { Hombres } \\ \text { Esfera doméstica } & \text { Esfera pública } \\ \begin{array}{l}\text { Trabajo de la casa } \\ \text { (no remunerado) }\end{array} & \begin{array}{l}\text { Trabajo } \\ \text { remunerado }\end{array} \\ \begin{array}{l}\text { Barreras para el } \\ \text { ejercicio del poder }\end{array} & \begin{array}{l}\text { Barreras para } \\ \text { expresar } \\ \text { emociones }\end{array} \\ \begin{array}{l}\text { Limitaciones para } \\ \text { el ejercicio de la } \\ \text { autonomía }\end{array} & \begin{array}{l}\text { Limitaciones para } \\ \text { la comunicación } \\ \text { igualitaria }\end{array}\end{array}$

La discriminación se reproduce al avalar como naturales los estereotipos que no permiten a mujeres y hombres desarrollarse según sus intereses y potencialidades. La discriminación se perpetra tanto por lo que se hace como por lo que no se hace: falta de pensamiento crítico, cerrar opciones sin explorar posibilidades, no tomar en cuenta otras perspectivas, etc.

Dos de las formas más fuertes en el proceso de discriminación es no contar con modelos alternativos a seguir y la persistencia en el uso de un lenguaje patriarcal ${ }^{1}$.

Propuesta feminista: La discriminación debe ser erradicada de las relaciones interpersonales. Todas las personas merecen la estimulación social para poder desarrollar sus capacidades. La diferencia entre público y privado es parte de la ideología patriarcal. La familia debe ser responsabilidad compartida. Todo trabajo debe ser reconocido socialmente y las mujeres -y los hombres- deben tener acceso tanto a los ámbitos de la casa como a los públicos.
Uno de los avances más importantes del feminismo es su cuestionamiento de la esfera privada, sosteniendo LO PRIVADO ES POLÍTICO.

\section{Control del cuerpo de las mujeres}

Una de las formas más fuertes de control en el patriarcado se produce por medio de normas tendientes a ejercer presión sobre la utilización del cuerpo de las mujeres, así, establecen la siguiente dicotomías:

$\begin{array}{ll}\begin{array}{l}\text { Cuerpo de las } \\ \text { Mujeres }\end{array} & \begin{array}{l}\text { Cuerpo de los } \\ \text { Hombres }\end{array} \\ \text { Complacer } & \text { Controlar } \\ \text { Bellos } & \text { Fuertes } \\ \text { Suaves } & \text { Rudos } \\ \text { Seguir normas } & \begin{array}{l}\text { Crear normas } \\ \text { de control } \\ \text { "morales" en lo } \\ \text { sexual y } \\ \text { reproductivo }\end{array} \\ & \begin{array}{l}\text { mujeres en lo } \\ \text { sexual y } \\ \text { reproductivo }\end{array}\end{array}$

Aspectos particularmente relevantes en el proceso de control patriarcal son la sexualidad y la reproducción. Cuando se produce una trasgresión a las normas las mujeres son culpabilizadas por la agresión que reciben.

Propuesta feminista: Derecho de las mujeres a conocer y decidir sobre su cuerpo.

\section{Violencia}

En el patriarcado los hombres -como forma de mantener el control- son sistemáticamente educados para la violencia, 
mientras que a las mujeres se les reprime cualquier expresión de ésta. Así los niños son entrenados por medio de modelos agresivos, tales como los que se presentan con frecuencia en películas y videojuegos.

Propuesta feminista: Nadie debe ser educado para ejercer la violencia, se debe propiciar el diálogo y la tolerancia.

\section{El feminismo ante la ciencia}

Afirma María Ángeles Durán que "la ciencia ha sido construida desde el poder y el poder ha puesto a la ciencia a su servicio, y... también se ha construido de espaldas a la mujer y a menudo en contra de ella." (Durán, María Ángeles, 1982, p. 9).

Como es el caso de todos los grupos que han sido discriminados, no es hasta que logran abrirse espacios y traspasar las "puertas cerradas" -en este caso del conocimiento científico- y que se producen cambios sustanciales hacia el trato igualitario. Por los milenios de opresión, el sexismo no se supera fácilmente, sino que requiere de reflexión y cuestionamiento permanente de las estructuras y procesos elaborados desde los tronos.

Hace casi veinte años María Ángeles Durán (1982) indicaba en su artículo "Liberación y Utopía: la mujer ante la ciencia” que el trabajo científico está inmerso en el contexto social, en forma tal que el sexismo se presentaba en:

- La selección de los temas de investigación.

- $\quad$ La selección de las perspectivas teóricas.

- La aceptación de los resultados de la investigación.

- La elección de los criterios de evaluación.
- La difusión de los resultados.

- $\quad$ El reconocimiento profesional y académico.

- Las normas y prácticas sociales de los colectivos creadores de ciencia o conocimiento especializado, tales como Escuelas, Academias, Universidades, Sociedades Científicas, etc.

En resumen, Durán plantea que el sexismo se encuentra enraizado en todas las etapas de la producción científica. Antes de la Década de la Mujer (1975-85) el sexismo se consideraba "natural" y no recibían, como en épocas más recientes, la crítica o asombro social aquellos que lo ejercían. Ahora, sin embargo, es más difícil encontrar posiciones discriminatorias presentadas con claridad, sino que, en su mayoría, toman formas encubiertas y particularmente solapadas, en forma tal que el sexismo debe buscarse con mayor perspicacia.

En este proceso de búsqueda y apertura, la creación de instancias universitarias específicas, tal como el Programa Interdisciplinario en Estudios de Género en 1987, base del Centro de Investigaciones de la Mujer aprobado en 1999, así como de la Maestría Regional en Estudios de la Mujer - proyecto conjunto de la Universidad de Costa Rica y la Universidad Nacional- en 1993, han facilitado pasos importantes para el cambio hacia la equidad. (Ver González Suárez, Mirta y Guzmán Stein, Laura, "Los Estudios de la Mujer: Desafiando el pasado, Construyendo el futuro", 1994).

La denuncia acerca de la discriminación de las mujeres en la academia ha sido sostenida a nivel nacional e internacional. Antecedentes particularmente relevantes para la tarea son los estudios de Florence Denmark et al., (1988) quienes analizan las diversas formas que la discriminación por género toma en la investigación en psicología. Edna Acosta Belén y Christine Bose 
(1991) recogen propuestas inclusivas de las mujeres en la historia y en la academia, tema analizado ese mismo año en España, cuyos resultados fueron publicados con el título: "Los estudios sobre la mujer: de la investigación a la docencia" (Bernis, Cristina et al., 1991), donde se hace referencia a las ciencias de la salud, sociología, lingüística e historia.

El informe elaborado, en marzo de 1999, por profesores y autoridades académicas del MIT (Massachusetts Institute of Technology), reconoce claramente la existencia de discriminación contra mujeres académicas en la Escuela de Ciencias. "El documento expone una discriminación extendida, aunque involuntaria (sic), en las áreas de contratación, premios, promociones e inclusión de las mujeres en los comités de asignación de recursos... Al finalizar el estudio, este grupo define a la discriminación como un patrón de actitudes y suposiciones, no reconocidas pero poderosas, que trabajan sistemáticamente en contra del profesorado femenino"2 (Goldberg, Carey, 1999).

$\mathrm{Y}$, si esto sucede en una institución "modelo" y afecta a sus profesoras, cuánto más discriminadas estarán las que no tienen voz en los procesos de investigación que las afectan. Tal vez uno de los ejemplos más patéticos ha sido la utilización, por décadas, de la episiotomía, hasta que a fines del siglo pasado la Organización Panamericana de la Salud señaló que, después de haberse investigado al respecto, consideraba a tal cirugía innecesaria, inconveniente y dañina en la mayor parte de los casos. Pero, hasta ese estudio reciente, miles de mujeres -a pesar de sus reiteradas quejas- debieron sufrir un procedimiento que, sin prueba científica, se asumió como válido y correcto.

En el Foro Regional de la UNESCO, que se llevó a cabo en Bariloche, Argentina, en octubre de 1998, se plantearon es- trategias hacia la equidad incluyéndose las siguientes para las políticas y programas de promoción: " 1 . Incorporar a las mujeres en las actividades de la ciencia y la tecnología..., propiciar la promoción de mujeres a puestos de liderazgo, proporcionándoles las mismas oportunidades que a los hombres, favorecer la educación de la mujer a nivel superior y promover la participación de las mujeres en la toma de decisiones por parte de las agencias de ciencia y tecnología, a fin de que los apoyos económicos se otorguen exclusivamente con fundamentos académicos y no de sexo, como las estadísticas muestran que sucede actualmente" (UNESCO, 1998, p. 25).

Tal como lo plantea María Arrilaga "La omisión de la óptica femenina del currículo básico universitario perpetúa una economía patriarcal, donde tanto hombres como mujeres llevamos las de perder. Una visión incompleta de la realidad es una visión parcializada. Una visión parcializada limita nuestra conciencia, nuestro sentido de justicia, nuestras posibilidades de adquirir la mejor información posible, el vuelo de nuestra imaginación, nuestra capacidad humana, en fin” (Arrilaga, María, 1992, p. 1).

\section{El sexismo en la academia}

El sexismo se encuentra presente en todos los niveles educativos (ver González Suárez, Mirta, "El sexismo en la educación. La discriminación cotidiana”, 1990). En la educación superior el sexismo se encuentra presente en sus tres ámbitos principales: la docencia, la investigación y la acción social ${ }^{3}$.

En la ponencia presentada en el seminario organizado por la Universidad de Puerto Rico (ver Azize, Yamila, 1992) con el título "Cuestionar lo incuestionable: el sexismo en el proceso educativo" la 
autora hace referencia a cuatro aspectos centrales en los que se manifiesta discriminación hacia las mujeres en la educación superior:

- La escogencia de carrera, por parte del estudiantado.

- $\quad$ El desarrollo científico androcéntrico.

- Las actitudes sexistas en el proceso enseñanza-aprendizaje.

- La distribución de puestos directivos, docentes, de investigación y otros.

(Ver González Suárez, Mirta, 1992).

\section{Escogencia de carrera:}

La escogencia de carrera se realiza frecuentemente, no por las habilidades, intereses o aptitudes, sino por la presión social, que induce a definirse por alternativas estereotipadas, a saber, carreras vinculadas con lo históricamente doméstico para las mujeres y con lo externo al hogar para los hombres. Se ha observado que esta última escogencia se relaciona también con la posibilidad de acceder a trabajos mejor remunerados y más reconocidos socialmente. Dichas decisiones sobre el futuro laboral implican una gran pérdida social, además de personal, e indican la importancia de proporcionar al estudiantado los elementos necesarios para cuestionar los estereotipos internalizados, en forma tal que puedan escoger de acuerdo con sus potencialidades y preferencias.

\section{El desarrollo científico androcéntrico:}

La ciencia, como todos los quehaceres, tiene el sello histórico de sus creadores. Si la teoría contiene elementos sexistas, necesariamente éstos se reflejarán en los pasos a seguir, las conclusiones y recomendaciones.
Ejemplos claros son aquellas posturas patriarcales que:

a) Asumen que las diferencias entre hombres y mujeres se derivan de bases genéticas o fisiológicas, por lo que son innatas y determinantes,

b) Desdeñan el papel del aprendizaje y la cultura,

c) Consideran irrelevante la capacidad de toma de decisiones así como del conocimiento del medio de las personas afectadas,

d) Validan los roles tradicionales de hombres y mujeres,

e) Suponen que las formas de opresión son culpa de las mismas mujeres. (Ver González Suárez, 1996).

Por otro lado, teorías que se jactan de "objetividad", pero basan sus resultados en mediciones que no consideran la influencia social y el contexto, igualmente pueden inducir a resultados tendenciosos, estableciéndose relaciones causales inadecuadas al considerar como naturales las condiciones discriminatorias actuales. Así Benbow y Stanley, (1980) concluyeron una investigación indicando que los puntajes en matemática de las jóvenes eran inferiores a los de los varones. Esta afirmación, sin análisis de las condiciones sociales, fue la base para que en los medios de comunicación se planteara que "Los hombres tienen inherentemente mayor capacidad matemática que las mujeres" (Benbow y Stanley citado en González Suárez, (1990) ${ }^{4}$.

Alternativas teóricas como las señaladas tienen en común la discriminación hacia las mujeres pero además proveen a la sociedad de una "base científica" para continuar con dicha práctica. 
Durán (1984, p. 31 y 32) propone un proceso de reflexión de cada ciencia, sintetizándolo en los siguientes puntos:

- Recuperación crítica de la historia de la disciplina.

- Explicitación y crítica de la metateoría subyacente.

- Crítica de los elementos sexistas encubiertos en la teoría.

- Crítica de los elementos sexistas encubiertos en los conceptos o en su operativización.

- Crítica de los efectos sexistas derivados de la utilización de algunos procedimientos o técnicas específicas de observación, medición o recolección de datos.

- $\quad$ Crítica de la organización social en la producción de la ciencia o disciplina.

- $\quad$ Crítica de la incongruencia o fosilización de los conocimientos sobre la mujer contenidos en la disciplina, así como de los criterios de evaluación de los mismos.

- $\quad$ Crítica al uso de los conocimientos proporcionados por la disciplina en la vida y en la práctica profesional.

- Explicitación de las demandas de nuevos conocimientos que puede satisfacer la disciplina.

- Reflexión crítica sobre los medios con que se puede contribuir al rechazo de conocimientos sesgados y a la potenciación de los nuevos conocimientos libres de sexismo que se solicitan en la ciencia.

La participación de los grupos discriminados en el quehacer científico implica una reconstrucción de las categorías "que son relevantes para sí y no lo fueron para sus predecesores" (Durán, 1984, p. 19). Afirma Durán que la creación de un grupo de investigación especializado requiere de liberarlos de las tareas cotidianas de producción, es decir, implica la reestructuración de la división social del trabajo.
La realidad es que las teorías androcéntricas deben ser reemplazadas por teorías científicas, hacia la búsqueda de un mayor bienestar tanto social como individual, superando las diversas formas de discriminación y en el contexto del respeto a los derechos humanos.

\section{Actitudes sexistas en el proceso enseñanza-aprendizaje}

El patriarcado es parte de las relaciones sociales y por lo tanto se presenta también en las relaciones académicas. Tal afirmación, sin embargo, en general no ha sido asumida como real a nivel de las carreras universitarias, donde es generalmente omitida en los análisis curriculares y evaluaciones de todo tipo.

La Ley contra el Hostigamiento Sexual en el Empleo y la Docencia (Ley 7476, 1995) junto con la creación del Reglamento de la Universidad de Costa Rica y la Comisión especial contra el Hostigamiento Sexual, dio forma a procesos para dejar atrás penosas agresiones, que anteriormente no se enfrentaban debidamente.

A continuación se mencionarán algunas formas -unas más sutiles que otrasen que se presentan actitudes sexistas en las relaciones interpersonales en el ámbito universitario:

Minimización: Las mujeres parecen inexistentes, tanto en el currículo como en los materiales educativos. No se cuestiona el sexismo como un factor social integrado a toda actividad.

Androcentrismo: Las interpretaciones y análisis "científicos" se presentan dentro de un contexto masculinizado.

Negación de la emoción: Estas son consideradas como parte del estereotipo 
femenino, por lo tanto se las excluye como inadecuadas dentro del ámbito académico.

Negación de otras perspectivas: No se aceptan visiones diversas sobre una situación. Por ej. Se obliga a realizar acciones que no aportan al desarrollo académico, sino que han sido una práctica tradicional o ritos de pasaje, tal como torturar sin obtener de ello un mayor conocimiento científico o pedagógico.

Exclusión de actividades: No se consideran las particularidades de las mujeres y en lugar de alternativas se promueven exclusiones, tales como paseos a los que deben asistir obligatoriamente, aún cuando el conocimiento pudiera ser adquirido de otras formas, perjudicando así a madres en período de lactancia o con hijos pequeños.

Valoración diferenciada: Evaluaciones diferenciadas en la participación de hombres y mujeres, por ej. Se valora el comportamiento agresivo en los varones, calificándose de competitivo y apropiado, no así en las mujeres, donde se percibe como grosero e inadecuado.

Segundo plano: En igualdad de condiciones se escoge usualmente a los candidatos hombres. Por ej. A nivel de asistentes, profesores o en la participación en clase.

Polos estereotipados: Las expectativas de comportamiento son diferentes para hombres y mujeres y se les recompensa de acuerdo a lo anterior.

Paternalismo: La comunicación con las mujeres no se realiza a nivel adulto, sino en forma condescendiente, etc.

Comentarios sexistas: Se presentan expresiones abiertamente derogatorias, como por ej. En mi clase no aprueban las mujeres, porque no se dedican lo suficiente.
Normalización de lo inadecuado: Se presenta ante la clase un tratamiento, que da a entender que lo normal es que los profesores sean sexistas, por ej. Yo no soy machista, como la mayoría, aunque sí me gusta que las mujeres se den a respetar...

Ocultación: Falta de posibilidades o alternativas de defensa ante las agresiones. No se considera aceptable recurrir a instancias oficiales o solicitar apoyo.

Hostilidad abierta y encubierta: Bromas hirientes sobre el cuerpo de las mujeres, comentarios derogatorios sobre el vestido, miradas inapropiadas, resentimiento sobre el sexo o la escogencia sexual del estudiantado.

Agresión física: Desde el besuqueo indeseado, toqueteo hasta la violación.

\section{Distribución de puestos directivos, docentes, de investigación y otros}

Un elemento omitido frecuentemente en el análisis del sexismo en la Universidad es el hecho de que el personal docente también se encuentra inmerso en el sistema patriarcal, y, por lo tanto, puede presentarse como un modelo del mismo.

¿Cuántas rectoras ha habido en la Universidad de Costa Rica, por ejemplo?

La escogencia estereotipada de carrera a la que se ha hecho referencia anteriormente se reproduce en el profesorado, donde las mujeres se concentran en áreas tradicionalmente relacionadas con lo doméstico.

El acceso a títulos académicos es igualmente diferenciado, y en la Universidad de Costa Rica el porcentaje del personal docente femenino es menor a medida que 
aumenta el nivel de los títulos. De hecho las posibilidades de proseguir su educación son menores para las mujeres. Ante el interés de continuar un postgrado, en la mayoría de los casos el profesional es avalado por su familia, la que interpreta sus estudios como un deseo de superación, el que, posteriormente le reparará una mejor posición económica y social.

En el caso de las mujeres tal situación no es tan obvia, ya que necesariamente debe convencer a su familia -sobre todo si tiene pareja e hijos- de que sus estudios no interferirán con el tiempo que les dedica. En general se aprecia su interés como una motivación personal y hasta egoísta, en detrimento de sus funciones de esposa y madre.

Una situación similar se percibe en los posibles viajes al exterior, alternativa usual para obtener un doctorado. Si el interesado es un hombre, después de tomada la decisión, su pareja usualmente la aceptará como medio de progreso social, incluso cuando deba abandonar su trabajo o carrera, y aún en el caso que no tenga una misión específica en el país al que se dirigen. Es conocido como muchas profesoras universitarias han acompañado a sus maridos al exterior, ocupándose primordialmente de la casa y los hijos. Sin embargo, en el caso de que una mujer desee salir del país para continuar estudiando, deberá en primer lugar convencer a su familia al respecto y no es usual que su pareja se adapte a una función de soporte. Más aún, la posibilidad de rechazo si el viaje pudiera interferir con el trabajo o carrera del esposo o compañero es muy alta.

Por otro lado, aquellas profesionales que no tienen pareja estable y viajan con sus hijos o solas deben continuar ocupándose de la doble jornada, lo que necesariamente implica un desgaste mayor.
Hemos señalado algunos de los aspectos que hacen a las mujeres más difícil su promoción académica. Tales circunstancias repercuten de igual manera en el logro de puestos directivos. Además las mujeres no cuentan con un número de modelos de poder similar a los de los hombres ya que históricamente han existido escasas posibilidades de práctica en la lucha por el poder fuera del hogar.

Por otro lado, la aceptación de nuevas responsabilidades pueden entrar en conflicto con la vida familiar: lo que para un hombre es un logro que merece un aplauso para una mujer puede ser causa de reproches.

Con respecto a los rasgos de personalidad, la competencia, la toma de iniciativas y conductas de liderazgo pueden ser percibidas como valores positivos en un hombre, pero en una mujer los mismos hechos pueden interpretarse como agresivos y "poco femeninos", ya que no coinciden con la imagen "maternal y protectora" socialmente esperada. Esta última, sin embargo, puede considerarse, por otro lado como un rasgo de incapacidad directiva. Tales atributos, evidentemente, tienen relación con las posibilidades de acceso a puestos directivos.

Ante el ingreso de las mujeres al medio universitario muchos colegas continuarán su vida profesional dentro de los mismos esquemas patriarcales, esperando que ellas se adapten a los mismos. Ejemplo de lo anterior son las reuniones fuera de horarios usuales, las que para los hombres, constituyen una grata posibilidad de intercambiar experiencias dentro de un ambiente más festivo. Para muchas mujeres, sin embargo, constituyen una experiencia indeseada, especialmente para aquellas que preferirían estar con su familia en los escasos momentos que tienen, tales como los fines de semana. 
Estas reuniones informales, son de hecho muy importantes en el esquema de poder, ya que el compartir implica camaradería y a su vez una mayor posibilidad de influencia y de conocimiento de hechos importantes. La no integración a estas actividades puede incluso contribuir a la pérdida de importantes oportunidades laborales. Por tal razón muy pocas académicas se atreven a plantear que prefieren hacer otra actividad fuera del horario laboral, ya que esto podría ser interpretado como falta de interés profesional.

Igualmente hay que considerar que las fórmulas de evaluación docente también recogen los estereotipos y sesgos mencionados: estudiantes que evaluarían a una mujer como insensible y rígida, podrían considerar a un profesor con características similares, como de alto nivel académico.

Es frecuente cuestionar la autoridad de las mujeres, en diversas formas sutiles. Demostraciones de poder son percibidas como amenazas, mientras que la suavidad y condescendencia son apreciadas como debilidad. Ambos casos son evaluados como poco o muy femeninos, siendo considerados igualmente inapropiados para el ejercicio del poder. Ante tales disyuntivas es lógico desarrollar un sentimiento de inseguridad, especialmente ante la posibilidad de ocupar puestos directivos.

Tales circunstancias usualmente requieren, tal como se ha mencionado, de una aceptación familiar de las nuevas condiciones, pero además suscitan un proceso de autoevaluación: ¿Seré yo la mejor opción? Podré desempeñarme adecuadamente? ¿Deberé cambiar mi forma de vida? ¿Tendré problemas en el hogar? ¿Cómo me tratarán los colegas? Es muy probable que ante un estricto cuestionamiento de las características personales y sociales, mu- chas personas, en especial, aquellas con un espíritu crítico desarrollado, se decidirán por no presentar su candidatura, perdiéndose así personas valiosas que probablemente faciliten el desarrollo universitario más que aquellas que no cuestionan su derecho a acceder a tales puestos.

Indica Brown Zikmund (1988) que uno de los problemas específicos de las profesionales es que quieren cumplir con todo en forma excelente. Surge así la idea de la "supermujer" quien es una excelente directora, profesora, investigadora, madre, esposa, amante y ama de casa, además de contribuir con su comunidad. La realidad es que el ingreso de las mujeres al trabajo fuera del hogar no ha sido paralelo a la redistribución social de funciones. Ante lo anterior existen dos alternativas: adaptarse a las actuales reglas de juego del poder aumentando los niveles de stress e insatisfacción, o modificar dichas reglas. Esta última alternativa es la única viable para la salud mental de la población.

Es importante tomar conciencia no sólo de las formas claras de sexismo sino también de aquellas más encubiertas en el tratamiento de las académicas:

Negar status y autoridad: Recurrir a otras personas, no aceptar las decisiones tomadas.

Criticar con base a características específicas: forma de peinarse, vestirse, modales, vida sexual, etc.

Lenguaje patriarcal: Utilizar un lenguaje sexista o nombrar a los hombres por su título académico y a las mujeres por su nombre o diminutivo de éste, etc.

Invisibilidad: No reconocer la contribución de las mujeres. 
Relegar funciones: Las mujeres sirven café, toman notas, etc. mientras los hombres leen las decisiones tomadas, coordinan, etc.

Restricción: No permitir el acceso de información a las mujeres, formar núcleos cerrados que se reúnen en espacios especiales, etc.

Discriminación múltiple: Se presentan tantos tipos de discriminación que no es factible determinar cuál es la más relevante; edad, grupo étnico, discapacidad, etc.

Diferenciación conductual: Se evalúa en forma diferenciada el comportamiento según el género.

\section{Conclusiones y recomendaciones}

Como opción propositiva en la construcción de una sociedad mejor, la universidad debe realizar un esfuerzo sustancial para enfrentar la discriminación y promover alternativas de relaciones humanas adecuadas.

En la academia es incorrecto discriminar porque:

- $\quad$ Contradice la misión de la universidad.

- $\quad$ Es éticamente reprobable.

- Desperdicia valiosos recursos humanos restringiendo su aporte social.

- Promueve un desarrollo científico sesgado.

Pero además de lo anterior es ilegal, como lo indica, entre otras, la Convención contra todas las Formas de Discriminación hacia la Mujer y la Ley para la Promoción Social de la Mujer. No se trata entonces de una opción de conducta sino de un mandato.
He aquí una importante contradicción, ya que hemos afirmado que el patriarcado nos ha inducido a creer que diversas formas de discriminación son naturales y correctas. ¿Cómo resolver este dilema? Por un lado por medio de estudios feministas, cuyo contenido crítico logren, paso a paso el objetivo central de esta teoría, a saber, la revelación y cambio de las estructuras patriarcales. Por otro, revisar sistemáticamente los resultados de las acciones. Tampoco se resuelve la situación, como se creía hace unos años, al tratar a todas las personas por igual. Comportarse en forma igual cuando las condiciones son diversas puede constituirse en una forma más de discriminación. El objetivo más bien es la equidad, es decir, tratar de forma tal que se logre la igualdad de oportunidades.

Como personas educadas dentro de una sociedad patriarcal hombres y mujeres somos portadores de modelos sexistas, a la vez que procuramos el cambio de los mismos. Esta dialéctica tradición-mundo nuevo que debemos enfrentar día a día, requiere de un doble esfuerzo para su superación, ya que somos, a la vez, partícipes y cuestionadores de los esquemas imperantes.

Se ha comprobado que por lo internalizado y profundo del modelo patriarcal, las buenas intenciones no son suficientes. Se requiere de un esfuerzo sistemático, para develar las formas abiertas y encubiertas de la discriminación, y el fruto se percibirá en los resultados concretos.

Tanto en la enseñanza, como en la investigación y la acción social, se requiere preguntar:

¿Cómo están representados los intereses de las mujeres en el proceso? 
¿Son las teorías, métodos y procedimientos discriminatorios?

¿Es la estructura de poder equitativa o primordialmente la toma de decisiones se concentra en los hombres?

¿Qué grupos quedan invisibilizados en la producción científica?

¿Se valora socialmente la participación y la tolerancia por la diferencia, o, se considera natural promover y perpeturar la discriminación?

¿Se promueve una sociedad con igualdad de oportunidades?

No olvidemos las metas establecidas en nuestro Estatuto Orgánico de una Universidad que es la conciencia crítica de la sociedad y la promotora del cambio social para un futuro mejor.

Desde el feminismo, algunas de las propuestas centrales en la academia son las siguientes,

- $\quad$ Promover la equidad de género.

- Valorar las experiencias, necesidades y perspectivas de mujeres $\mathrm{y}$ hombres.

- Desarrollar la autonomía de las personas, especialmente de las mujeres, sobre las actividades, su cuerpo, etc.

- Develar las estructuras de poder y fomentar la participación de las mujeres en la toma de decisiones.

- Desarrollar, especialmente en los hombres, la no violencia, el respeto y la responsabilidad familiar compartida.

- Desarrollar, especialmente en los hombres, el valor humano de las emociones como forma de comunicación humana.

- Utilizar un lenguaje no discriminatorio.
- Desarrollar la tolerancia por la diferencia.

- Cambiar las relaciones agresivas por alternativas respetuosas para la resolución de conflictos.

- Impulsar valores humanos tales como cooperación, ternura, apoyo, colaboración, convivencia, independencia, etc.

- Propiciar la igualdad de oportunidades entre hombres y mujeres.

- Evaluar por medio de resultados concretos el proceso de cambio social hacia la equidad.

Para finalizar, algunas preguntas básicas, cuyas respuestas deberían presentarse públicamente a la entrada de cada unidad académica.

1. ¿Cómo se presenta la distribución de ingreso por sexo? ¿Qué hace la Escuela/Facultad para atraer a jóvenes de ambos sexos por medio de una política de igualdad de oportunidades?

2. ¿Hay diferencia en las notas finales obtenidas por hombres y mujeres? ¿A qué se debe esta diferencia?

3. ¿Cuántos hombres y mujeres se gradúan en cada carrera?

4. ¿Cómo se presenta la distribución del profesorado por sexo? (Interinazgo y en propiedad).

5. ¿Cuál es la distribución de puestos de poder por sexo?

6. ¿Cuál es la participación de los estudiantes de ambos sexos en los puestos de asistencias, dirigencia estudiantil, etc.?

7. ¿Se han analizado los contenidos patriarcales en teorías, investigación, acción social, etc.? ¿A quienes 
benefician los resultados de los trabajos realizados?

8. ¿Qué formas encubiertas y específicas presenta el sexismo en su unidad académica?

9. ¿Qué medidas específicas se han tomado para superar el sexismo?

10. ¿Qué resultados concretos han tenido tales medidas?

\section{Notas}

1. Por su extensión no se presentará en esta ocasión un análisis de este punto, que puede ser revisado en diversas publicaciones tales como en Calvo, Yadira (1984 y 1990)". Literatura, mujer y sexismo" y "A la mujer por la palabra" Macaya, Emilia (1992) "Cuando estalla el silencio: para una lectura femenina de textos hispánicos" y Demonte, Violeta (1991). "Sobre la expresión lingüística de la diferencia”.

2. El subrayado es de la autora.

3. En esta oportunidad se enfatizará el análisis a nivel general, especialmente en la docencia, ya que la autora ha presentado una ponencia específica sobre la investigación (Ver González Suárez, Mirta, 1999 "Sesgos solapados: el sexismo en la investigación ") y no se cuenta todavía con suficientes datos en la acción social.

4. Para discriminación de las mujeres en matemática ver Chavarría, Silvia, (1994).

\section{Referencias bibliográficas}

Acosta-Belén, Edna y Bose, Christine. (Ed). "Perspective and Resources. Integrating Latin American and Caribbean Women into the Curriculum and Research" Albany: CELAC/IROW. 1991.

Amorós, Celia. "Historia de la Teoría Feminista" Madrid: Instituto de Investigaciones Feministas de la Univer- sidad Complutense de Madrid y Dirección General de la Mujer. 1994.

Arrilaga, María. "Un currículo balanceado: para no defraudar el ejercicio de la docencia" en Azize, Yamila (1992) Hacia un Currículo no sexista. Universidad de Puerto Rico, Colegio Universitario de Cayey. 1992.

Azize, Yamila. "Hacia un Currículo no sexista". Universidad de Puerto Rico, Colegio Universitario de Cayey. 1992.

Batres, Gioconda. "Del ultraje a la esperanza”. San José: ILANUD. 1997.

Calvo, Yadira. "Literatura, mujer y sexismo". San José: Editorial Costa Rica. 1984.

. "A la mujer por la palabra" Heredia: Editorial Universidad Nacional. 1990.

Carcedo, Ana y Delso, Ana Isabel y equipo de CEFEMINA. (Sin fecha) "Mujeres hacia el 2000. Deteniendo la violencia”. San José: CEFEMINA.

Claramunt, Cecilia "Casitas Quebradas. El problema de la violencia doméstica en Costa Rica”. San José: EUNED. 1997.

. "Explotación sexual en Costa Rica: niños, niñas y adolescentes hacia la prostitución”. San José: UNICEF. 1998.

Chavarría, Silvia "Matemática sesgada por género" en Revista de Ciencias Sociales número 65, p. 127-132. 1994.

Denmark, Florence, Russo, Nancy, Frieze, Irene y Sechzer, Jeri. "Guidelines for Avoiding Sexism in Psychological Research" American Psychologist, July, vol. 43, no. 7, p. 582-585. 1988. 
Demonte, Violeta. "Sobre la expresión lingüística de la diferencia". En: Los Estudios sobre la Mujer. De la Investigación a la Docencia Madrid: Edigrafos. 1991.

Durán, María Ángeles. "Liberación y utopía: La mujer ante la ciencia" Madrid: Ed. Akal. 1982.

Goldberg, Carey. "MIT Admits Discrimination against Female Professors" New York Times, martes 23 de marzo de 1999.

González Suárez, Mirta. (Ed.) "Estudios de la Mujer: Conocimiento y Cambio”. San José: EDUCA. 1988.

"El sexismo en la educación. La discriminación cotidiana". San José: Editorial Universidad de Costa Rica. 1990.

. "Cuestionar lo incuestionable: El sexismo en el proceso educativo" en Azize, Yamila (1992) Hacia un $\mathrm{Cu}$ rrículo no sexista. Universidad de Puerto Rico, Colegio Universitario de Cayey. 1992.

González Suárez, Mirta y Guzmán Stein, Laura. "Los Estudios de la mujer en Costa Rica: Desafiando el pasado, Construyendo el futuro" en Revista de Ciencias Sociales de la Universidad de Costa Rica, n. 65, sept. 1994, p. 7-16. 1994.

. "Feminismo en Costa Rica? Testimonios, Reflexiones, Ensayos". San José: Editorial Mujeres. 1995.

"Jaque al Rey: de la psicología patriarcal a la psicología feminista". Revista Costarricense de Psicología número 24, Año 12, 1996, p. 11-29. 1996.
- "Necesidades de Investigación en Estudios de la Mujer en Centroamérica”. Actualidades en Psicología, Instituto de Investigaciones Psicológicas de la Universidad de Costa Rica, Volumen 13, número 95. 1997.

. "Sesgos solapados: sexismo en la investigación”. Ponencia (en prensa) al Seminario Regional de Historia, Universidad de Costa Rica. 1999.

Guzmán, Laura y Pacheco, Gilda. (Compiladoras) "Estudios Básicos de Derechos Humanos IV". San José: Instituto Interamericano de Derechos Humanos. 1996.

Harris, Judith. "The Nurture Assumption" Psychological Review, 1995, vol. 102, n. 3, p. 458-489. 1995.

Macaya, Emilia. "Cuando estalla el silencio: para una lectura femenina de textos hispánicos”. San José: Editorial de la Universidad de Costa Rica. 1992.

Madrigal, Johnny et al. "Factores relacionados con el embarazo no deseado en Costa Rica". San José: Asociación Demográfica Costarricense. 1990.

Molleda, José, Negrini, Ana y Osorio, Gisselle "Análisis crítico de la concepción freudiana de la mujer". Tesis en Psicología, Universidad de Costa Rica. 1982.

Programa Mujeres Adolescentes. "Programa de Acción a favor de las Adolescentes y Jóvenes Madres en Costa Rica, Honduras y Nicaragua”. Disco Compacto. San José: Unión Europea, Programa de Mujeres Adolescentes y Consejo de Integración Social. 1999. 
Polster, Miriam. "Eve's Daughters: The Forbidden Heroism of Women" San Francisco: Jersey-Bass Publishers. 1992.

Statham, Ann, Richardson, Laurel y Cook, Judith. "Gender and University Teaching" Albany: State University of New York Press. 1991.
UNESCO "Informe de las representantes de la UNAM al Foro Regional UNESCO" en Supercuerdas, Boletín para la mujer en la ciencia. Número 10, año 1999, p. 23-27. 1998.

Vargas, Maureen. "La mujer en la administración educativa desde una perspectiva de género: Historia y Poder". San José: CMF. 1997. 